K. Pravin Kashyap, Odelu Ojjela*, and Samir Kumar Das

\title{
MHD slip flow of chemically reacting UCM fluid through a dilating channel with heat source/sink
}

https://doi.org/10.1515/nleng-2018-0036

Received February 21, 2018; revised August 6, 2018; accepted September 30, 2018.

Abstract: The present article explores the effects of uniform heat source and first order destructive chemical reaction on an upper convected Maxwell fluid through an expanding or contracting channel with the porous slip condition at the upper plate. It is assumed that the fluid is sucked or injected through the upper plate. The temperature and concentration at the plates is maintained constant. Using suitable similarity transformations, nonlinear coupled ODEs are developed from the governing PDEs. The subsequent ODEs are converted into a first order system and integrated via shooting method. The effect of various prominent parameters on heat, flow and mass transfer characteristics are studied in detail through graphs and tables. The present results suggest that the presence of chemical reaction and heat source yields in the reduction of concentration and of the enhancement of temperature the fluid. It is also observed that the wall expansion shows an increasing effect on the radial velocity component, but the slip parameter exhibits an opposing effect. The viscous case has been studied as a special case where the present results are found to be close to the earlier ones. The flow of such nonlinear viscoelastic fluids has important applications in separation processes like petroleum and medical industries.

Keywords: Upper convected Maxwell fluid, porous slip, mixed convection, shooting method, chemical reaction

\footnotetext{
*Corresponding Author: Odelu Ojjela, Department of Applied Mathematics Defence Institute of Advanced Technology (Deemed University), Pune-411025, Maharashtra, India,

E-mail: odelu@diat.ac.in, odelu3@yahoo.co.in

K. Pravin Kashyap, Samir Kumar Das, Department of Applied Mathematics Defence Institute of Advanced Technology (Deemed University), Pune-411025, Maharashtra, India
}

\section{Introduction}

The expanding and contracting boundaries have applications in many practical situations such as of filtration, the regression of grains in the course of combustion of solid propellant, binary gas diffusion, artificial dialysis, the flow of blood, circulation of blood and air during the respiration process, separation processes of other membranes. Berman [1] considered the 2-D flow of a Newtonian fluid through stationary walls with same permeability and obtained an analytical solution. This study has been extended by Majadalini et al. [2] by coupling the injection and suction with wall motion. He studied the role of expansion wall ratio of a viscous laminar flow between two weakly permeable surfaces with same permeability which enables the fluid to flow in the course of expansion or contraction. The solutions were obtained by using both analytical and numerical procedures and the results were compared graphically. Majadalini and Zhou [3] extended the problem for large and moderate suction or injection flows in contracting or expanding channels with a membrane closing one end of the channel. For the same problem, Boutros et al. [4] developed Lie group solutions for Re, wall expansion and compared these solutions with the numerical solutions. Asghar et al. [5] developed an exact solution by using the laws of conservation and symmetry methods to reduce to the PDE to second order ODE and hence find solutions to the problems like Majadalini et al. [2]. Later, Asghar et al. [6] accomplished a solution with Adomain Decomposition Method for small Reynolds number and wall expansion ratios and compared the results with [2] and [4]. Hence a wide range of solution procedures were developed. In many metallurgical and chemical engineering processes $1^{\text {st }}$ order chemical reaction has an important role, Subramanyam et al. [7] employed a parameter to study the generative and destructive chemical reactions in expanding porous channels. The presence of temperature and concentration gradients in the channel induces the convection process in the channel. So authors [8-17] have considered the combined effects of chemical reactions like autocatalyis and porous slip conditions along with mixed convection to understand the flow properties. 
The flow of non-Newtonian fluids have attracted many researchers as most of the real world fluids are nonNewtonian. A large number of models were developed to analyze the properties like viscoelasticity, time independent and time dependent viscosities. Jambal et al. [18] studied the heat transfer and flow in circular ducts and parallel plates for a power law fluid by taking both dissipation due to the viscosity and the heat conduction of the fluid and analyzed the flow characteristics by using finite difference method. Odelu and Naresh [19] have studied the chemical reaction effects on a micropolar fluid confined in between an expanding or contracting porous channel. Chen and Zhu [20] have obtained an analytical solution for Couette-Poiseuille flow of Bingam fluids confined in a porous channel with velocity slip on the boundaries. Srinivasacharya et al. [21] obtained the profiles of velocity and temperature distributions for a streamlined flow of a couple stress fluid in porous expanding or contracting channel by employing quasilinearization method. Makinde and Chinyoka [22] considered a dusty fluid to study the transient MHD flow and heat transfer in a parallel channel with the Navier slip condition at the boundaries and obtained solution by using semi implicit finite difference scheme. Abbas and Sheikh [23] studied the effects of homogenous - heterogeneous reactions on a ferrofluid with flow above a flat plate with a stagnation point by assuming a slip condition on the boundary. They followed the non-linear slip condition suggested by Thompson and Troian [24] which is a function of shear rate at the boundary and their results revealed that the velocity and concentration increase with slip parameters. HAM solutions for a micropolar fluid between expanding/contracting porous plates with different permeabilities has been obtained by Si et al. [25]. Later, Xinhui et al. [26] extended the problem by considering the slip velocity based on Beavers and Joseph slip condition [27] but with an assumption of equal permeability at the plates. Shojaeian and Ali [28] studied the entropy generation and convective heat transfer of non-Newtonian and Newtonian fluids confined in between parallel plates by considering boundary conditions due to slip effect. Ahmed et al. [29] have analyzed the laminar nanofluid flow through the dilating permeable channel and used similarity transformations to reduce the flow field equation into non-linear ODE. The solution has been obtained by using Galarkin, Runge Kutta Fehelberg schemes and a comparison between the methods has been presented.

A systematic framework for the rheological behaviour of rate type of fluids has been developed by Oldroyd [30] and he later studied some steady flows of general elastic viscous fluids [31]. Zahorski [32], in his book "Mechanics of Viscoelastic fluids", has discussed the tensor theoretical representations along with rheological experimental studies. He also explained the fundamentals of mechanical principles of viscoelastic fluids and its relation to continuum in modern mechanics. Nasar et al. [33] have noticed that the shear thinning rate of the viscosity might cause a reduction in the normal velocity of Oldroyd-b fluid through a stretching channel. Si et al. [34] analysed the asymmetric viscoelastic flow through an expanding or contracting porous channel and obtained a solution by using HAM. Bhatti and Abbas [35] analysed the slip effects on the creeping flow of a Jefferey fluid under the action of an external magnetic field and discussed the effects for nonNewtonian and Newtonian cases. Kalpana and Sumit [36] extended this study for Jeffrey nanofluid and studied the effect of thermal radiation, viscous dissipation and magnetic field effects. By considering the Rivlin Ericksen tensors, grade fluids like second grade and third grade models have been developed [37]. Due to the importance of chemical reaction in many branches of chemical and biological engineering several authors [38-40] have incorporated the concentration equation along with the momentum equations. Hayat et al. [41] studied the magnetic field effects on a chemically reacting third grade fluid above a stretching sheet using HAM and analysed the velocity, skin friction, concentration and local Sherwood number profiles with respect to various parameters. Satya Narayana and Harish Babu [42] have observed that by enhancing the magnetic field strength, the longitudinal velocity can be decreased and it has an opposite effect with relaxation parameter of a Jeffrey fluid. They have also found that increasing heat source would enhance the temperature and chemical parameter decreases the concentration for a steady flow above a stretching sheet. Middleman [43] explained the implementation of upper convected derivative in the Maxwell model which provides a balance between physical reality and necessary mathematical simplicity for the numerical solution of practical problems. AlizadehPahlavan and Kayvan [44] inspected the effects of magnetic and elastic forces for an unsteady flow of UCM fluid over a stretching sheet and observed that the they have decreased effects on the flow. The effects of various parameters on temperature and velocity profiles in a MHD UCM fluid flow over a stretching sheet in the presence of a heat sink or source were analysed by Mukhopadhyay [45] by using a shooting technique. Vajravelu et al. [46] analysed the effect of chemical reaction on the MHD flow of an UC Maxwell fluid above a porous surface by using a Keller box numerical technique and observed that the order of the chemical reaction increases the diffusion boundary layer thickness. Hayat and Awis [47] considered a 3-D flow of UC 
Maxwell fluid above a stretching sheet and inspected the effect of the Deborah number on the velocity components. Bilal et al. [48] investigated 3-D flow of an UCM fluid with nanoparticals and studied the effects of elasticity on flow, heat and boundary properties. Nandy [49] studied the effect of unsteady, velocity slip and Maxwell parameters on Nusselt number, heat, flow, skin friction, concentration, Sherwood numbers and showed that they have considerable effect for an UCM fluid over a permeable shrinking surface with nanoparticles. Choi et al. [50] have analyzed the 2-D flow of upper convected Maxwell fluid in a porous surface channel using both $4^{\text {th }}$ order Runge Kutta integration scheme and power series. Their observation suggested that at a constant Reynolds number the longitudinal velocity at the center line decreases for the higher Deborah number and it has a flattening effect on axial velocity for high Reynolds numbers for a fixed Deborah number. Hayat and Abbas [51] found HAM solutions for the 2-D boundary layer flow of a UC Maxwell fluid in a porous channel. Hayat et al. [52] have considered the action of radiation on MHD UCM fluid flow through a porous medium and obtained solution using HAM. Beg and Makinde [53] have analysed the species transfer of a UCM fluid flow through a Darcian high permeability channel at high Derborah numbers by using 6th order Runge Kutta method.

In the present article, a mixed convective flow of UCM fluid confined in a contracting or expanding porous channel with slip conditions is explored. The effect of heat source and chemical reaction on the concentration, heat transfer and the velocities is studied. The effect of various parameters on the temperature, concentration and velocity profiles is analysed and presented in the form of graphs. The axial velocity for the Newtonian case has been studied and the obtained results are close to the ones in the literature $[2,4,6]$.

\section{Nomenclature}

t Time (s)

$V_{1}$ Suction/injection velocity at upper wall $(\mathrm{m} / \mathrm{s})$

a(t) Distance from the origin to the upper wall (m)

$B_{0}$ Magnetic flux density (Tesla)

T Temperature (Kelvin)

$\mathbf{T}_{2}$ Temperature at the upper wall

$\mathbf{T}_{1}$ Temperature at the lower wall

C Concentration (moles)

$\mathbf{C}_{1}$ Concentration at the lower wall

$\mathbf{C}_{2}$ Concentration at the upper wall

u Velocity component in $\mathrm{x}$-direction $(\mathrm{m} / \mathrm{s})$

P Fluid pressure $(\mathrm{Pa})$ g Acceleration due to gravity $\left(\mathrm{m} / \mathrm{s}^{2}\right)$

v Velocity component in y-direction $(\mathrm{m} / \mathrm{s})$

c Specific heat at constant temperature $\left(\mathrm{J} \mathrm{kg}^{-1} \mathrm{~K}^{-1}\right)$

$\mathbf{Q}_{H}$ Heat source/sink

$\mathbf{k}_{r}$ Chemical reaction rate (s-1)

D Mass diffusivity $\left(\mathrm{m}^{2} / \mathrm{s}\right)$

k Thermal conductivity (Watt $/ \mathrm{m} / \mathrm{K}$ )

$\sigma_{1}$ Dimensionless constant which depends on the pore size of the permeable material

$\mathbf{k}_{1}$ Permeability of the porous medium (Darcy)

$\dot{n}_{A}$ Mass transfer rate

Wi Wiessenberg number $\frac{\beta V_{1}}{a}$

Re Permeation Reynolds number, $\frac{\rho V_{1} a}{\mu}$

Ha Hartmann number,

Ec Eckert number,

Gr Grashof number (thermal),

Gc Grashof number (solutal),

Sh Sherwood Number, $\frac{\dot{n}_{A}}{a v\left(C_{2}-C_{1}\right)}$

Pr Prandtl number,

Sc Schmidt number,

$\mathbf{K}_{r}$ Non dimensional chemical reaction parameter, $\frac{k_{r} a^{2}}{v}$

$\mathbf{H}_{s}$ Non dimensional heat source/sink parameter, $\frac{Q_{H} a^{2}}{k}$

Sl Non dimensional Slip parameter $\frac{\sqrt{k_{1}}}{a \sigma_{1}}$

\section{Greek Notations}

$\mu$ Fluid viscosity $(\mathrm{mPa} / \mathrm{sec})$

$\rho$ Fluid density $\left(\mathrm{Kg} / \mathrm{m}^{3}\right)$

$\lambda$ Dimensionless y coordinate,

$\xi$ Dimensionless axial variable,

$v$ Kinematic viscosity $\left(\mathrm{m}^{2} / \mathrm{s}\right)$

$\sigma$ Electric conductivity (Seimens)

$\beta$ Maxwell parameter (s)

$\alpha$ Wall expansion ratio,

$\beta_{T}$ Thermal expansion coefficient $\left(\mathrm{K}^{-1}\right)$

$\beta_{C}$ Solutal expansion coefficient $\left(\mathrm{Mol}^{-1}\right)$

\section{Problem formulation}

Consider an unsteady flow of upper convected Maxwell fluid in an expanding or contracting semi-infinite porous channel where the plates are placed at $\mathrm{Y}=0$ and $\mathrm{Y}=\mathrm{a}$. One end of the channel is closed by a stretching sheet or membrane which stretches or contracts as the distance between the plates, $\mathrm{a}(\mathrm{t})$ changes. The $\mathrm{X}$ and $\mathrm{Y}$ axes are taken along and perpendicular to the axial flow direction with a constant magnetic field in the Z- direction as shown in the Fig. 1. It is assumed that the fluid is constantly sucked 


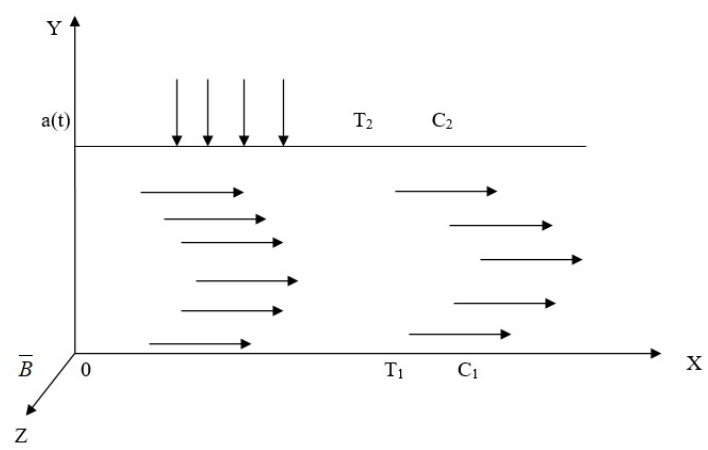

Fig. 1: The schematic representation of UCM fluid through expanding/contracting plates.

or injected from the upper plate with a velocity $\mathrm{V}_{1}$, the temperature and concentration at the plates are constantly maintained at $\mathrm{T}_{2}, \mathrm{C}_{2}$ and $\mathrm{T}_{1}, \mathrm{C}_{1}$. As convective heat transport in porous channels due to Ohmic heating and buoyancy forces have a major part in applications of heat exchangers and nuclear reactors, we have considered the effect of mixed convection in the present model. The governing equations for the system is given by the equations (1)(5).

$$
\begin{gathered}
\nabla \cdot q=0 \\
\frac{\partial \vec{q}}{\partial t}+(\vec{q} \cdot \nabla) \vec{q}=-(\nabla P+\nabla \cdot S)+\mu \nabla^{2} \vec{q} \\
-J \times B+\rho g\left(\beta_{T}\left(T-T_{1}\right)+\beta_{C}\left(C-C_{1}\right)\right) \\
S+\beta\left(\frac{d S}{d t}+\vec{q} \cdot \nabla S-(\nabla \vec{q})^{T} \cdot S-S \cdot \nabla \vec{q}\right) \\
=\mu\left(\nabla \vec{q}+(\nabla \vec{q})^{T}\right) \\
\rho c\left[\frac{\partial T}{\partial t}+(\vec{q} \cdot \nabla) T\right]=k \nabla^{2} T+\frac{J^{2}}{\sigma}+Q_{H}\left(T-T_{1}\right) \\
\frac{\partial C}{\partial t}+(\vec{q} \cdot \nabla) C=D \nabla^{2} C-K_{r}\left(C-C_{1}\right)
\end{gathered}
$$

By following Mukhopadyay [45] and Hayat and Awis [47], the equations (6) - (10) are formulated which describe the continuity, momentum, energy and concentration of the current problem in a Cartesian coordinate system.

$$
\frac{\partial u}{\partial x}+\frac{\partial v}{\partial y}=0
$$

$\rho\left[\frac{\partial u}{\partial t}+u \frac{\partial u}{\partial x}+v \frac{\partial u}{\partial y}+\beta\left(u^{2} \frac{\partial^{2} u}{\partial x^{2}}+v^{2} \frac{\partial^{2} u}{\partial y^{2}}+2 u v \frac{\partial^{2} u}{\partial x \partial y}\right)\right]=$ $-\frac{\partial P}{\partial x}+\mu \nabla^{2} u-\sigma B_{0}^{2} u+\rho g \beta_{T}\left(T-T_{1}\right)+\rho g \beta_{C}\left(C-C_{1}\right)$

$$
\begin{gathered}
\rho\left[\frac{\partial v}{\partial t}+u \frac{\partial v}{\partial x}+v \frac{\partial v}{\partial y}+\beta\left(u^{2} \frac{\partial^{2} v}{\partial x^{2}}+v^{2} \frac{\partial^{2} v}{\partial y^{2}}+2 u v \frac{\partial^{2} v}{\partial x \partial y}\right)\right] \\
=-\frac{\partial P}{\partial y}+\mu \nabla^{2} v-\sigma B_{0}^{2} v \\
\rho c\left[\frac{\partial T}{\partial t}+u \frac{\partial T}{\partial x}+v \frac{\partial T}{\partial y}\right]=k\left(\frac{\partial^{2} T}{\partial x^{2}}+\frac{\partial^{2} T}{\partial y^{2}}\right) \\
+\sigma B_{0}^{2}\left(u^{2}+v^{2}\right)+Q_{H}\left(T-T_{1}\right) \\
\frac{\partial C}{\partial t}+u \frac{\partial C}{\partial x}+v \frac{\partial C}{\partial y}=D\left(\frac{\partial^{2} C}{\partial x^{2}}+\frac{\partial^{2} C}{\partial y^{2}}\right)-k_{r}\left(C-C_{1}\right)
\end{gathered}
$$

Where $\mathrm{P}$ is pressure, $\beta$ is the relaxation coefficient of Maxwell fluid, $\mathrm{Q}_{H}$ is heat source/ sink parameter, $\mathrm{D}$ is diffusivity coefficient and $\mathrm{k}_{r}$ is the chemical reaction parameter. The theory and experiments of Beavers and Joseph [27] demonstrates the importance of the velocity slip effect due to the porous nature of the boundary where the gradient of external velocity is proportional to the slip velocity. Later, this theory has been mathematically justified by Saffman [54] and Mikelic and Jager [55] through statistical and analytical methods to show that the approximate condition at the interface given by Beavers, Joseph [27] and Saffman [54] is satisfied by velocity field for a laminar flow of a Newtonian fluid above a porous surface. The slip velocity due to Beavers and Joseph's condition is considered in the boundary conditions (11) along with temperature and concentration at the plates.

$$
\begin{aligned}
& T=T_{1}, \quad C=C_{1} u=0, \quad v=0, \quad \text { at } y=0 \\
& T=T_{2}, \quad C=C_{2} u=-\frac{\sqrt{k_{1}}}{\sigma_{1}} \frac{\partial u}{\partial y}, \quad v=-V_{1}, \quad \text { at } y=a
\end{aligned}
$$

Majdalini et al. [2] devoloped solutions for the generalised Proudman and Johnson's [56] equation by using the following similarity variables (12) for velocity and this work has been extended to study the temperature and concentration [19].

$$
u=\frac{v x}{a^{2}} F^{\prime} \quad v=-\frac{v}{a} F
$$

$$
T=T_{1}+\frac{v V_{1}}{a c}\left(\phi_{1}+\frac{x^{2}}{a^{2}} \phi_{2}\right) \quad C=C_{1}+\frac{\dot{n}_{A}}{a v}\left(g_{1}+\frac{x^{2}}{a^{2}} g_{2}\right)
$$

Using the above variables a similarity solution in time and space is derived for constant $\alpha$ 's which are specified by the value of initial value $\alpha=\frac{a \dot{a}}{v}=\frac{a_{0} \dot{a}_{0}}{v}$ where $a_{0}, \dot{a}_{0}$ are the initial distance and wall expansion ratios. By following Asghar et al. [6], instead of $\lambda$ and $t$, the function $F$ is redefined to be a function of $\lambda$ and $\alpha(\mathrm{t})$. By assuming $\alpha$ to be constant 
or quasi constant in time, we have the condition $\mathrm{F}_{\lambda t}=0$. The relation between $\mathrm{F}$ and $\mathrm{f}$ is given by $F(\lambda)=\operatorname{Re} f(\lambda)$. Thus we have developed nonlinear ODEs from the governing equations (13) - (17).

$$
\begin{aligned}
& f^{i v}\left(1-W i \operatorname{Re} f^{2}\right)=-\alpha\left(\lambda f^{\prime \prime \prime}+3 f^{\prime \prime}\right)+\operatorname{Re}\left(f^{\prime} f^{\prime \prime}-f^{\prime \prime \prime} f\right) \\
& -2 W i \operatorname{Re} f^{\prime \prime}\left(\left(f^{\prime}\right)^{2}+f f^{\prime \prime}\right)+H a^{2} f^{\prime \prime} \\
& -\frac{E c G r}{\xi \operatorname{Re}}\left(\phi_{1}^{\prime}+\xi^{2} \phi_{2}^{\prime}\right)-\frac{S h G c}{\xi \operatorname{Re}}\left(g_{1}^{\prime}+\xi^{2} g_{2}^{\prime}\right) \\
& \phi_{1}^{\prime \prime}=-\alpha \operatorname{Pr}\left(\phi_{1}+\lambda \phi_{1}^{\prime}\right)-\operatorname{Re} \operatorname{Pr}\left(f \phi_{1}^{\prime}\right)-H a^{2} \operatorname{Re} \operatorname{Pr}\left(f^{2}\right) \\
& -2 \phi_{2}-H_{S} \phi_{1} \\
& \phi_{2}^{\prime \prime}=-\alpha \operatorname{Pr}\left(3 \phi_{2}+\lambda \phi_{2}^{\prime}\right)+\operatorname{Re} \operatorname{Pr}\left(\phi_{2} f^{\prime}-f \phi_{2}^{\prime}\right) \\
& \quad-H a^{2} \operatorname{Re} \operatorname{Pr}\left(f^{\prime}\right)^{2}-H_{S} \phi_{2} \\
& g_{1}^{\prime \prime}=-\alpha S c\left(g_{1}+\lambda g_{1}^{\prime}\right)-\operatorname{Re} S c f g_{1}^{\prime}+K_{r} g_{1}-2 g_{2} \\
& g_{2}^{\prime \prime}=-\alpha S c\left(3 g_{2}+\lambda g_{2}^{\prime}\right)+\operatorname{Re} S c\left(2 f^{\prime} g_{2}-f g_{2}^{\prime}\right)+K_{r} g_{2}
\end{aligned}
$$

The boundary conditions on the velocities, temperature and concentration in terms of $f(\lambda), \phi_{1}(\lambda), \phi_{2}(\lambda), g_{1}(\lambda)$ and $g_{2}(\lambda)$ are given in (18).

$$
\begin{aligned}
& f=0, \quad f^{\prime}=0, \quad \phi_{1}=0, \quad \phi_{2}=0, \quad g_{1}=0, \\
& g_{2}=0 \text { at } 0 \\
& f=1, \quad f^{\prime l}=-\frac{f(1)}{S l}, \quad \phi_{1}=1 / E c, \quad \phi_{2}=0, \quad g_{1}=1 / S h \text {, } \\
& g_{2}=0 \text { at } 1
\end{aligned}
$$

For practical purposes, the non-dimensional functions of skin friction, local Sherwood and Nusselt numbers [46] are given by

$$
\begin{aligned}
& C_{f}=\frac{\mu}{\rho V_{1}^{2}} \frac{\partial u}{\partial y} \quad S h=\frac{-a}{\left(C_{2}-C_{1}\right)} \frac{\partial C}{\partial y} \\
& N u=\frac{-a}{\left(T_{2}-T_{1}\right)} \frac{\partial T}{\partial y}
\end{aligned}
$$

\section{Solutio $\mathrm{n}$ procedure}

A nonlinear system of first order equations (21) is formulated from equations (13)-(17). Shooting method is implemented by reducing the BVP to IVP and an algebraic nonlinear system. To integrate the IVP till $\lambda=1$, we need sufficient boundary conditions at $\lambda=0$. Hence, we assume guess slopes $\mathrm{as}_{1}, \mathrm{as}_{2}, \mathrm{as}_{3}, \mathrm{as}_{4}, \mathrm{as}_{5}, \mathrm{as}_{6}$ to form the initial conditions at the lower boundary (20).

$$
\begin{aligned}
& z_{1}=0 ; \quad z_{2}=0 ; \quad z_{3}=a s_{1} ; \quad z_{4}=a s_{2} ; \quad z_{5}=0 ; \\
& z_{6}=a s_{3} \text {; } \\
& z_{7}=0 ; \quad z_{8}=a s_{4} ; \quad z_{9}=0 ; \quad z_{10}=a s_{5} ; \quad z_{11}=0 ; \\
& z_{12}=a s_{6} \text {; } \\
& z_{1}=f \quad z_{5}=\phi_{1} \quad z_{7}=\phi_{2} \quad z_{9}=g_{1} \quad z_{11}=g_{2} \\
& z_{2}=f^{\prime} \quad z_{3}=f^{\prime /} \quad z_{4}=f^{\prime / I} \\
& z_{4}^{\prime}=f^{i v}=\left(-\alpha\left(\lambda z_{4}+3 z_{3}\right)+\operatorname{Re}\left(z_{2} z_{3}-z_{1} z_{4}\right)\right. \\
& -2 W i \operatorname{Re}\left(z_{2}^{2} z_{3}+z_{1} z_{3}^{2}\right)+H a^{2} z_{3} \\
& \left.-\frac{E c G r}{\xi \operatorname{Re}}\left(z_{6}+\xi^{2} z_{8}\right)-\frac{S h G c}{\xi \operatorname{Re}}\left(z_{(10)}+\xi^{2} z_{12}\right)\right) /\left(1-W i \operatorname{Re} z_{1}^{2}\right) \\
& z_{5}^{\prime}=z_{6}=\phi_{1}^{\prime} \\
& z_{6}^{\prime}=\phi_{1}^{\prime \prime}=-\alpha \operatorname{Pr}\left(z_{5}+\lambda z_{6}\right)-\operatorname{Pr} \operatorname{Re}\left(z_{1} z_{6}\right) \\
& -H a^{2} \operatorname{Pr} \operatorname{Re}\left(z_{1}\right)^{2}-H_{S} \phi_{1}-2 z_{7} \\
& z_{7}^{\prime}=\phi_{2}^{\prime}=z_{8} \\
& z_{7}^{\prime}=\phi_{2}^{\prime \prime}=-\alpha \operatorname{Pr}\left(z_{7}+\lambda z_{8}\right)+\operatorname{Pr} \operatorname{Re}\left(2 z_{2} z_{7}-z_{1} z_{8}\right) \\
& -H a^{2} \operatorname{Pr} \operatorname{Re}\left(z_{2}\right)^{2}-H_{S} z_{7} \\
& z_{9}^{\prime}=g_{1}^{\prime}=z_{10} \\
& z_{10}^{\prime}=g_{1}^{\prime \prime}=-\alpha S c\left(z_{9}+\lambda z_{10}\right)-S c \operatorname{Re} z_{1} z_{10}+K_{r} z_{9}-2 z_{11} \\
& z_{11}^{\prime}=g_{2}^{\prime}=z_{12} \\
& z_{12}^{\prime}=g_{2}^{\prime \prime}=-\alpha S c\left(3 z_{11}+\lambda z_{12}\right)+\operatorname{ReSc}\left(2 z_{2} z_{11}-z_{1} z_{12}\right) \\
& +K_{r} z_{11}
\end{aligned}
$$

The assumed slopes form a column vector 'as'. Due to the fast convergence of Newton Raphson scheme, the algebraic problem is formulated in vector form using the conditions at the upper boundary. This numerical problem is solved by using a suitable initial guess vector for 'as' and iterate the vector ' $\mathrm{y}$ ' to get the values at the upper boundary by using R-K $4^{\text {th }}$ order algorithm such that the algebraic problem is satisfied.

\section{Results}

The unsteady slip flow of mixed convective chemically reacting upper convected Maxwell fluid through an expanding or contracting channel with uniform heat source is investigated with numerical computations using a shooting approach. The effect of various prominent non dimensional parameters such as Heat source parameter $\left(\mathrm{H}_{S}\right)$ and non-dimensional chemical reaction parameter $(\mathrm{Kr})$, Weissenberg number (Wi), slip parameter (Sl), wall expansion/contraction parameter $(\alpha)$, Hartmann number (Ha) on non-dimensional temperature and species concentration distributions, heat and mass transfer coefficients, the 
Table 1: Effect of various parameters on skin friction, heat and mass transfer coefficients at $\mathrm{Ec}=1 ; \mathrm{Gr}=9 ; \mathrm{Re}=3 ; \mathrm{Ha}=2 ; \mathrm{Gc}=9 ;=0.632 \xi 5$; $\mathrm{Sh}=1 ; \mathrm{Sc}=0.95 ; \mathrm{Pr}=0.7$

\begin{tabular}{|c|c|c|c|c|c|c|c|c|}
\hline \multirow{2}{*}{\multicolumn{2}{|c|}{$\begin{array}{l}\text { Parameter } \\
\lambda=C\end{array}$}} & \multirow[b]{2}{*}{$\lambda=1$} & \multicolumn{2}{|c|}{ Skin Friction } & \multicolumn{2}{|c|}{ Heat transfer coefficient } & \multicolumn{2}{|c|}{ Mass transfer coefficient } \\
\hline & & & $\lambda=0$ & $\lambda=1$ & $\lambda=0$ & $\lambda=1$ & & \\
\hline \multirow{4}{*}{$\mathrm{Wi}=0.1 \alpha=1 \mathrm{Hs}=5 \mathrm{Kr}=0.2$} & \multirow{4}{*}{$\mathrm{SI}$} & 0.1 & 5.3752 & -3.1240 & 13.0219 & -7.9759 & 1.7270 & 0.0665 \\
\hline & & 0.2 & 4.8436 & -2.5091 & 12.6092 & -8.0988 & 1.7046 & 0.0752 \\
\hline & & 0.3 & 4.4921 & -2.0951 & 12.3536 & -8.2134 & 1.6896 & 0.0812 \\
\hline & & 2 & 3.2254 & -0.5480 & 11.5564 & -8.8576 & 1.6348 & 0.1045 \\
\hline \multirow{4}{*}{$\mathrm{Sl}=0.1 \mathrm{Wi}=0.1 \mathrm{Hs}=5 \mathrm{Kr}=0.2$} & \multirow{4}{*}{$\alpha$} & -1 & 5.1563 & -4.2102 & 8.4504 & -7.5839 & 1.0303 & 0.8186 \\
\hline & & 0 & 5.2149 & -3.6701 & 10.5025 & -7.8313 & 1.3474 & 0.4052 \\
\hline & & 1 & 5.3752 & -3.1240 & 13.0219 & -7.9759 & 1.7270 & 0.0665 \\
\hline & & 2 & 5.6556 & -2.5891 & 16.0874 & -8.0255 & 2.1687 & -0.2019 \\
\hline \multirow[t]{4}{*}{$\mathrm{Wi}=0.1 \alpha=1 \mathrm{Sl}=0.1 \mathrm{Kr}=0.2$} & \multirow{4}{*}{$\mathrm{H}_{S}$} & -4 & 5.6620 & -3.6514 & 4.4078 & -2.3290 & 1.7181 & 0.0711 \\
\hline & & 0 & 5.5750 & -3.5295 & 6.4846 & -3.9885 & 1.7196 & 0.0702 \\
\hline & & 2 & 5.5138 & -3.4233 & 8.2399 & -5.1772 & 1.7213 & 0.0692 \\
\hline & & 3 & 5.4758 & -3.3490 & 9.4462 & -5.9294 & 1.7227 & 0.0686 \\
\hline \multirow[t]{4}{*}{$\mathrm{Wi}=0.1 \alpha=1 \mathrm{Sl}=0.1 \mathrm{Hs}=5$} & \multirow{4}{*}{$\mathrm{Kr}$} & 0 & 5.3724 & -3.1199 & 13.0237 & -7.9756 & 1.7777 & 0.0186 \\
\hline & & 2 & 5.3988 & -3.1544 & 13.0086 & -7.9776 & 1.3476 & 0.4628 \\
\hline & & 4 & 5.4219 & -3.1788 & 12.9989 & -7.9785 & 1.0452 & 0.8463 \\
\hline & & 6 & 5.4422 & -3.1966 & 12.9926 & -7.9787 & 0.8253 & 1.1866 \\
\hline \multirow[t]{4}{*}{$\alpha=1 \mathrm{Sl}=0.1 \mathrm{Hs}=5 \mathrm{Kr}=0.2$} & \multirow{4}{*}{ Wi } & 0 & 5.3369 & -3.2303 & 12.9592 & -7.9934 & 1.7238 & 0.0678 \\
\hline & & 0.1 & 5.3752 & -3.1240 & 13.0219 & -7.9759 & 1.7270 & 0.0665 \\
\hline & & 0.15 & 5.4079 & -3.0602 & 13.0614 & -7.9649 & 1.7292 & 0.0657 \\
\hline & & 0.2 & 5.4519 & -2.9889 & 13.1078 & -7.9522 & 1.7384 & 0.0647 \\
\hline
\end{tabular}

Table 2: Comparison values of axial velocity in the case of Newtonian fluid at $\operatorname{Re}=5$ and $\alpha=0.5$

\begin{tabular}{ccccc}
\hline$\lambda$ & Majdalani et al. [2] & Asghar et al. [4] & Boutros et al. [6] & Present \\
\hline 0 & 1.536002 & 1.559474 & 1.556324 & 1.55947443 \\
0.05 & 1.531846 & 1.554822 & 1.551780 & 1.55482216 \\
0.1 & 1.519377 & 1.540888 & 1.538164 & 1.54088861 \\
0.15 & 1.498596 & 1.517743 & 1.515522 & 1.51774361 \\
0.2 & 1.469505 & 1.485503 & 1.483935 & 1.48550349 \\
0.25 & 1.432114 & 1.444331 & 1.443517 & 1.44433105 \\
0.3 & 1.386445 & 1.394435 & 1.394421 & 1.39443543 \\
0.35 & 1.332539 & 1.336071 & 1.336839 & 1.33607179 \\
0.4 & 1.270464 & 1.269540 & 1.271006 & 1.26954084 \\
0.45 & 1.200325 & 1.195188 & 1.197207 & 1.19518811 \\
0.5 & 1.122275 & 1.113403 & 1.115778 & 1.11340290 \\
0.55 & 1.036527 & 1.024617 & 1.027110 & 1.02461693 \\
0.6 & 0.943364 & 0.929302 & 0.931656 & 0.92930265 \\
0.65 & 0.843156 & 0.827971 & 0.829933 & 0.82797120 \\
0.7 & 0.736373 & 0.721170 & 0.722523 & 0.72117004 \\
0.75 & 0.623597 & 0.609480 & 0.610078 & 0.60948025 \\
0.8 & 0.505538 & 0.493513 & 0.493322 & 0.49351360 \\
0.85 & 0.383052 & 0.373909 & 0.373046 & 0.37390927 \\
0.9 & 0.257149 & 0.251330 & 0.250109 & 0.25133045 \\
0.95 & 0.129010 & 0.126461 & 0.125435 & 0.12646066 \\
1 & 0 & 0 & 0 & 0 \\
\hline
\end{tabular}

velocity components and the skin friction are studied in the domain $[0,1]$ and the results are shown with the help of tables and graphs.

The slip velocity due to Beavers and Joseph condition [27] effects the overall flow, temperature and concentration across the channel. The Figure 2 depicts the slip parameter's effect on the velocity components. It is noticed that the axial component decreases towards the middle and then it tends to increase towards the upper boundary, on the other hand the radial component gets reduced for increasing slip parameters. This is because of the slip velocity results in the reduction of the pressure gradient. The mass transfer rate and skin friction decrease at the lower plate and increase at the upper plate while the rate of heat transfer decreases at both the plates for higher slip parameter coefficients. Hence the heat transfer rates at the plates 


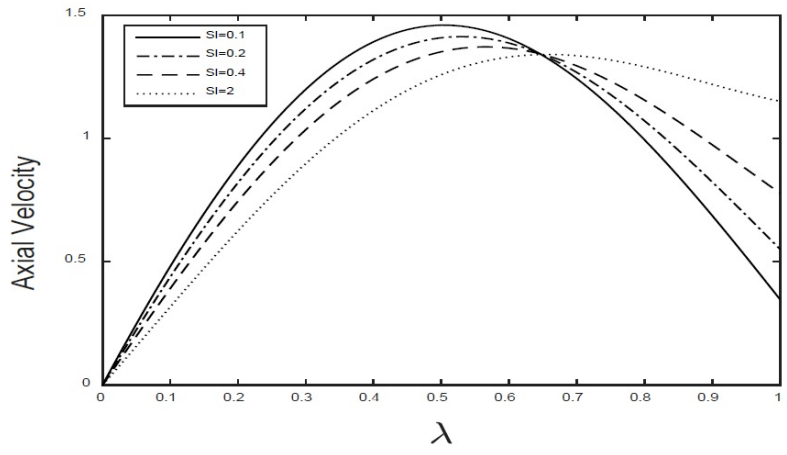

(i)

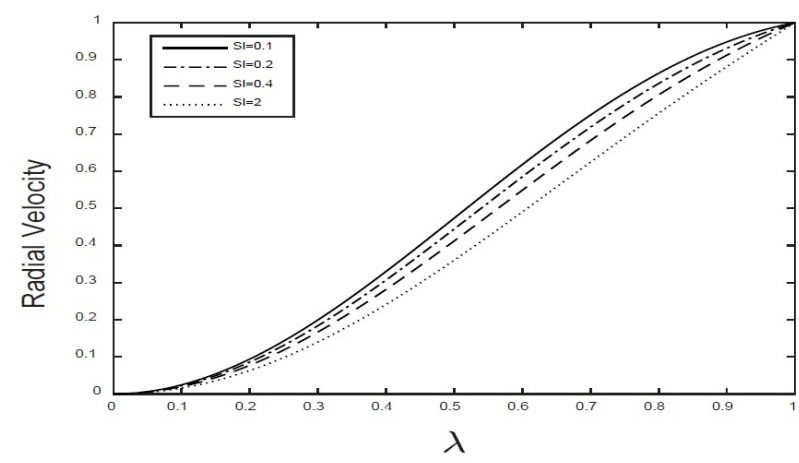

(ii)

Fig. 2: Slip parameter's effect on (iii) Axial velocity (iv) Radial velocity with $\mathrm{EC}=2 ; \mathrm{Sh}=2 ; \mathrm{Re}=3.4 ; \mathrm{Pr}=0.7 ; \mathrm{Gr}=11.56 ; \mathrm{Wi}=0.05 ; \mathrm{Ha}=$ $2 ; \mathrm{Gc}=11.56 ; \mathrm{Sc}=1.4 ;=0.6325 \xi ; \mathrm{H}_{S}=2 ; \mathrm{Kr}=0.2 . \alpha=0.1$

can be enhanced by choosing the porous surfaces with less slip parameters for the given parametric values.

The results for various values of expansion wall ratio i.e. temperature, concentration, longitudinal and radial velocities are portrayed in the Figure 3. The solid represents the contraction ratio and the dot dashed line, i.e. $\alpha=0$ represents the fixed boundary and the other two lines show the expansion of the channel. The temperature, concentration and the radial components are observed to be increasing for higher $\alpha$. The axial velocity is found to be increasing towards the middle of the channel and decreasing towards the upper boundary for increasing $\alpha$. From the Table 1 , it is observed that the skin friction increases at both the plates with $\alpha$ whereas the heat and mass transfer coefficients increase at the lower boundary on the contrary it decreases at the upper plate. This shows that the temperature in the channel and heat transfer rate at the upper plate enhance during the expansion of the channel.

The presence of heat source/sink in the channel is illustrated in the Figure 4 with temperature profiles. The solid line represents the heat sink, the dot dashed line represents the absence of the heat source in the present model. It is clear to see that the temperature is increas-

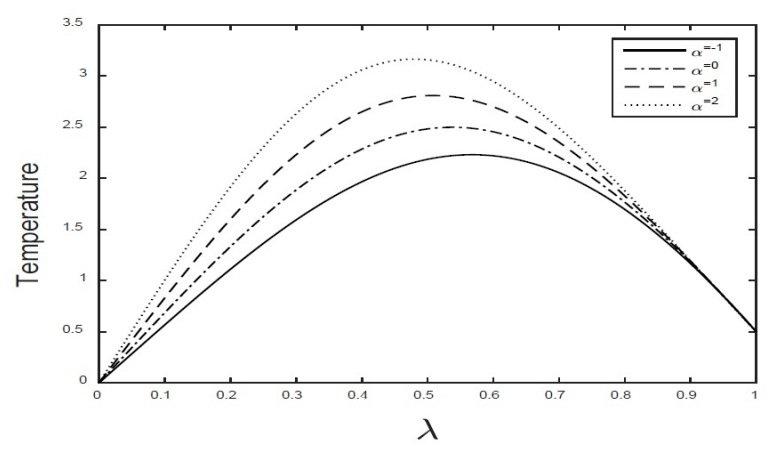

(i)

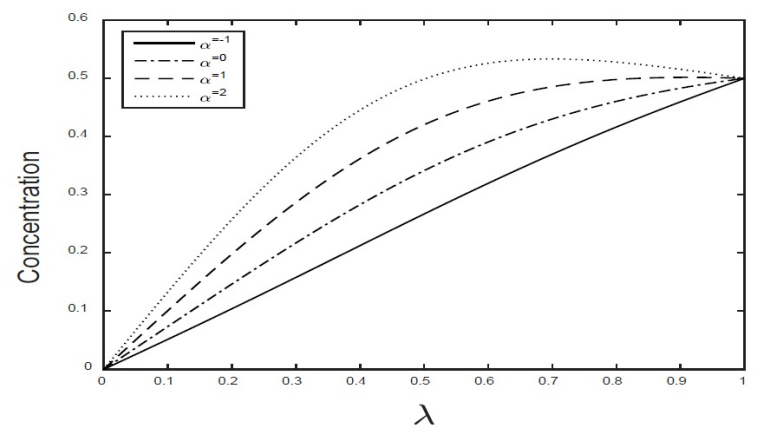

(ii)

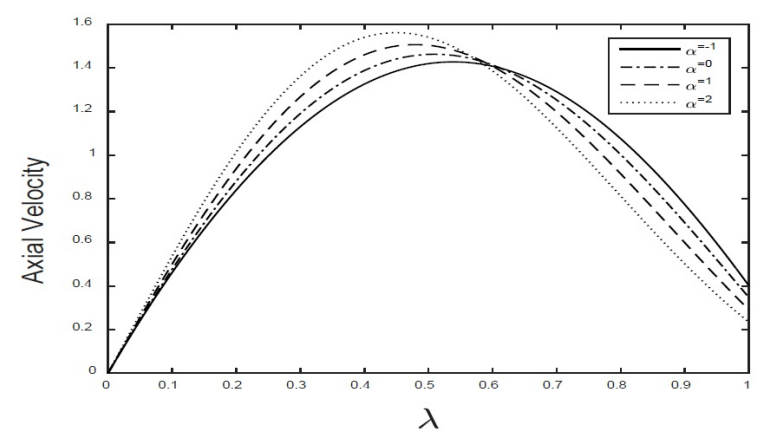

(iii)

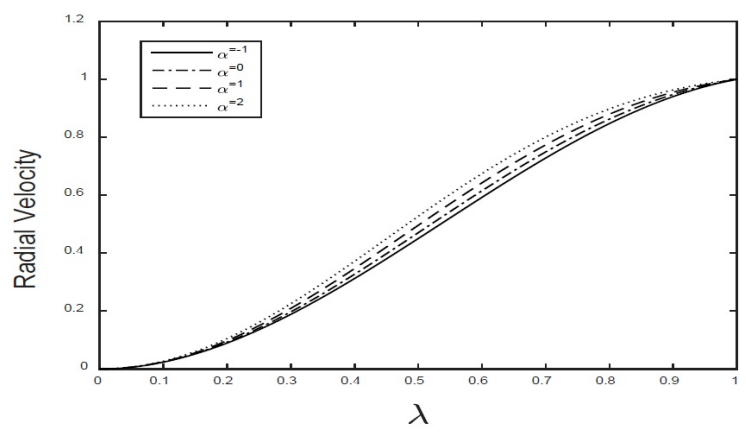

(iv)

Fig. 3: Effect of wall expansion/ contraction parameter on (i) Temperature (ii) Concentration (iii) Axial velocity (iv) Radial velocity with $\mathrm{Ec}=2 ; \mathrm{Sh}=2 ; \mathrm{Pr}=0.71 ; \mathrm{Re}=3 ; \mathrm{Wi}=0.05 ; \mathrm{Ha}=3 ; \mathrm{Gr}=9 ; \mathrm{Gc}=9 ; \mathrm{Sc}$ $=1.2 ;=0.632 \xi 5 ; \mathrm{H}_{S}=1 ; \mathrm{Kr}=0.4 ; \mathrm{Sl}=1$. 


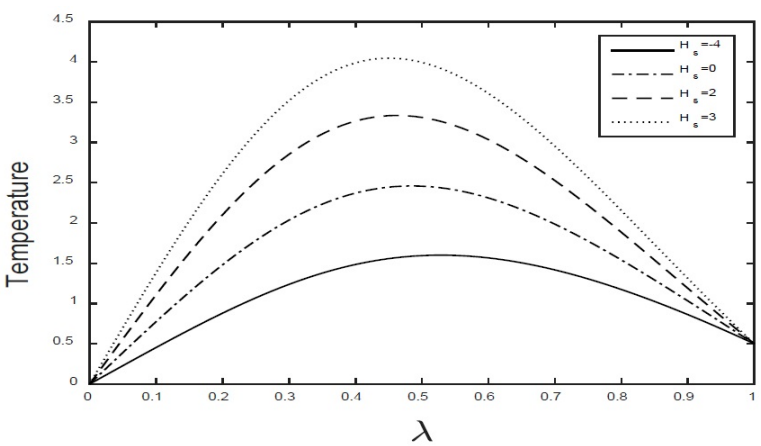

Fig. 4: Influence of Heat source parameter on Temperature with $\mathrm{Ec}=$ $2 ; \mathrm{Pr}=0.4 ; \mathrm{Sh}=2 ; \mathrm{Re}=4 ; \alpha=4 ; \mathrm{Sl}=1 ; \mathrm{Ha}=3 ; \mathrm{Wi}=0.2 ; \mathrm{Gr}=16 ; \mathrm{Gc}=$ $16 ; \mathrm{Sc}=1.5 ;=0.632 \xi 5 ; \mathrm{Kr}=4$.

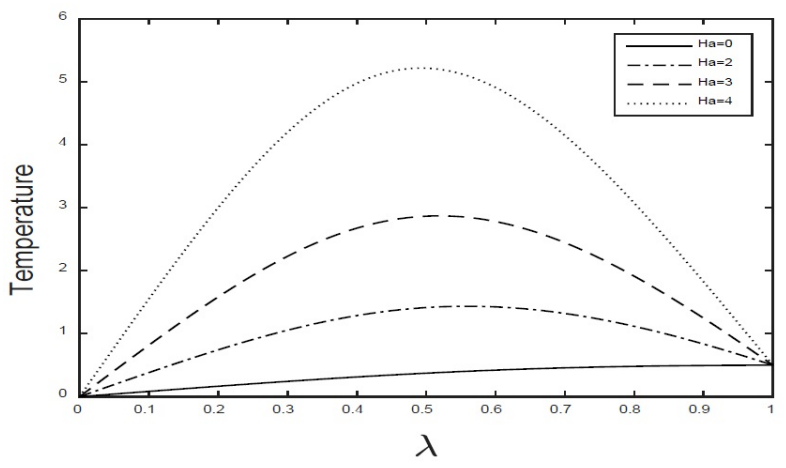

Fig. 5: Influence of Hartmann number on Temperature with $\mathrm{Ec}=2$; $\operatorname{Pr}=0.7 ; \operatorname{Re}=4 ; \mathrm{Wi}=0.05 ; \alpha=5 ; \mathrm{Gr}=16 ; \mathrm{Gc}=16 ; \mathrm{Sc}=1.5 ;=0.6325 \xi$; $\mathrm{Sh}=2 ; \mathrm{H}_{S}=0.5 ; \mathrm{Kr}=2 ; \mathrm{Sl}=1$.

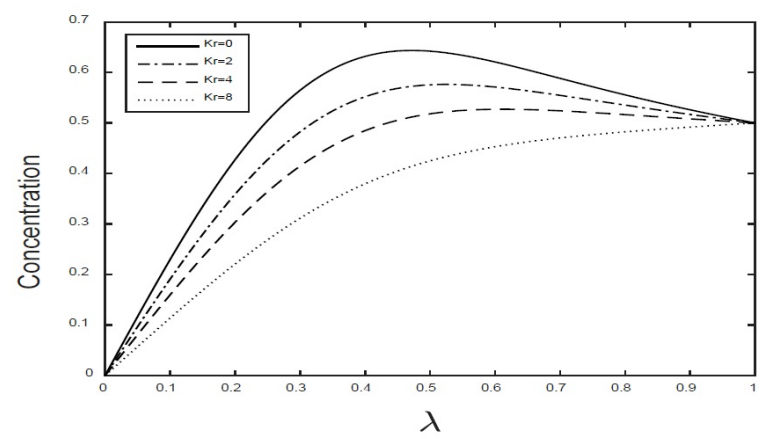

Fig. 6: Effect of chemical reaction parameter on Concentration with $\mathrm{Ec}=2 ; \mathrm{Pr}=0.4 ; \mathrm{Re}=5 ; \mathrm{Wi}=0.1 ; \alpha=4 ; \mathrm{Ha}=3 ; \mathrm{Gr}=25 ; \mathrm{Gc}=25 ; \mathrm{Sc}=$ $1.5 ;=0.632 \xi 5 ; \mathrm{Sh}=2 ; \mathrm{H}_{S}=1 ; \mathrm{Sl}=1$.

ing with heat source parameter. The increment in heat source parameter implies more heat production in the fluid and thus increasing the overall temperature. The heat and mass transfer coefficients increase at the lower and decrease at the upper boundaries, whereas the skin friction has an opposite pattern for higher heat source parametric values. The effect of Joule heating on the fluid due to the

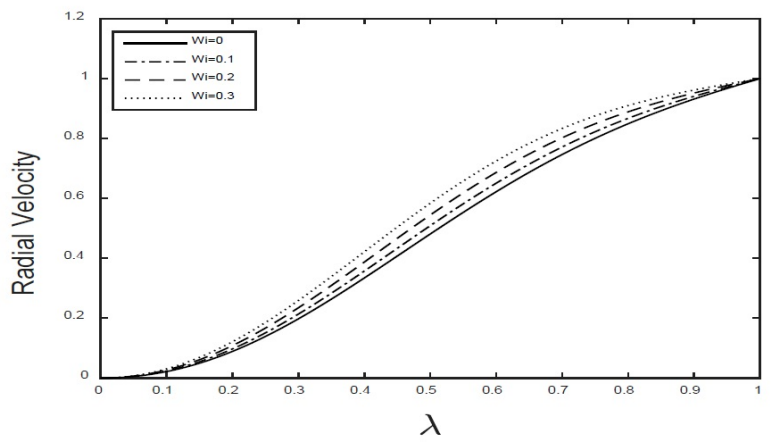

(i)

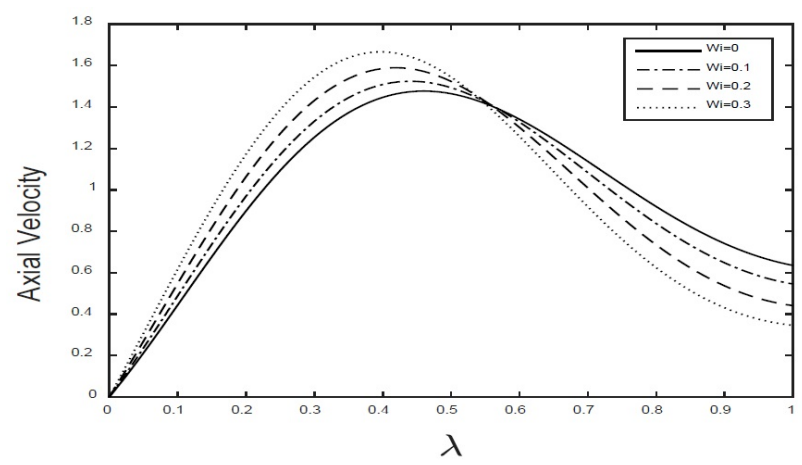

(ii)

Fig. 7: The influence of Wi on (i) Radial velocity (ii) Axial velocity with $\mathrm{Ec}=2 ; \mathrm{Pr}=0.7 ; \mathrm{Re}=3.4 ; \alpha=4 ; \mathrm{Ha}=3 ; \mathrm{Gr}=11.56 ; \mathrm{Gc}=11.56 ; \mathrm{Sc}=$ $1.4 ;=0.632 \xi 5 ; \mathrm{Sh}=2 ; \mathrm{H}_{S}=2 ; \mathrm{Kr}=0.2 ; \mathrm{Sl}=1$.

applied external magnetic field is depicted in Figure 5. Increasing magnetic field increases the Lorentz force on the fluid [57], Joule heating of the fluid is due to the resistivity of the fluid which has a natural property to raise its temperature under external magnetic field. The absence of magnetic field is represented by the solid line. The gradual increment of the temperature of the electrically conducting fluid due to increasing Hartmann numbers.

The Figure 6 shows the impact of chemical reaction on the heat, mass and flow characteristics. The solid line in the Figure 6 represents the absence of chemical reaction in the present problem. It is evident from the figure that the concentration decreases for higher chemical reaction parameters. As the chemical reaction is of destructive nature, increasing the chemical reaction parameter would inflate the reduction of concentration of the species across the channel. Apart from the concentration, the effect of $\mathrm{Kr}$ on the boundary properties is also studied. As chemical reaction parameter increases, the skin friction also increases at the lower boundary, but decreases at the upper one. The heat transfer coefficient decreases at both the boundaries and the mass transfer coefficient follows an opposite pattern of skin friction. Therefore, the presence of destructive 
chemical reaction decreases the concentration across the channel and increases the mass transfer rate at $\lambda=1$.

The Figure 7 shows the significance of elastic forces on the radial and axial components. The solid line represents the absence of Weissenberg effect i.e. Newtonian fluid. The radial velocity is increasing for increasing Wi values. Due to the domination of viscoelastic forces on inertial forces for Reynolds number less than 5 , it is observed that the longitudinal component increases towards the center and it declines up to the channel end. For increasing Weissengberg numbers, the enhancement of heat transfer and skin friction is noted at both boundaries while the mass transfer coefficients increase on the lower boundary and decrease at the upper one. The Newtonian case has been investigated and the attained non dimensional axial velocities are close to the existing ones $[2,4,6]$. The comparative results are shown in Table 2 .

\section{Conclusions}

The unsteady heat transfer and MHD flow of a chemically reacting mixed convective UCM fluid through expanding or contracting porous channel with a uniform heat source is studied numerically. The study concludes the following points.

- The fluid temperature is enhanced with the influence of the Joule heating effect due to the magnetic field and also with the heat source.

- The concentration distribution decreases for increasing chemical reaction parameter, whereas it increases with wall expansion ratio.

- The axial velocity profiles of the slip parameter and the Weissenberg number follow opposite trend.

- The radial velocity is increasing with the wall expansion ratio, while it is reduced due to the slip flow.

- Maxwell fluid and wall expansion ratio parameters show similar effects on skin friction and mass transfer rate at boundaries.

These results have possible applications in science and technology such as biorheology, food processing, fluid transport in biological organisms, polymer industry and petroleum industries. This study can be extended by considering other properties like varying viscosities and thermal conductivities across the channel which is very natural in the applications like separation of crude oil.
Acknowledgement: The authors are thankful to Vice Chancellor of Defence Institute of Advanced Technology (Deemed University) for his support in the current research. One of the authors (KPK) is grateful to the University Grants Commission, Government of India for providing Senior Research Fellowship (F.2-18/2012(SA-I)).

\section{References}

[1] A. S. Berman, Laminar flow in channels with porous walls, Journal of Applied Physics, 1953, 24(9), 1232-1235.

[2] J. Majdalani, C. Zhou, C. A Dawson, Two-dimensional viscous flow between slowly expanding or contracting walls with weak permeability, J Biomech., 2002, 35(10), 1399-1403.

[3] J. Majdalani, C. Zhou, Moderate-to-large injection and suction driven channel flows with expanding or contracting walls, ZAMM, 2003, 83(3), 181-196.

[4] Y. Z. Boutros, M. B. Abd-el-Malek, N. A. Badran, H. S. Hassan, Lie-group method solution for two-dimensional viscous flow between slowly expanding or contracting walls with weak permeability, Appl Math Model, 2007, 31(6), 1092-1108.

[5] S. Asghar, M. Mushtaq, A. Kara, Exact solutions using symmetry methods and conservation laws for the viscous flow through expanding-contracting channels, Applied Mathematical Modelling, 2008, 32(12), 2936-2940.

[6] S. Asghar, M. Mushtaq, T. Hayat, Flow in a slowly deforming channel with weak permeability: An analytical approach, Nonlinear Anal Real World Appl., 2010, 11(1), 555-561.

[7] A. Subramanyam Reddy, S. Srinivas, T. R. Ramamohan, Analysis of heat and chemical reaction on an asymmetric laminar flow between slowly expanding or contracting walls, Heat Transfer-Asian Research, 2013, 42(5), 422-443.

[8] R. Kumar, R. Kumar, S. A. Shehzad, M. Sheikholeslami, Rotating frame analysis of radiating and reacting ferro-nanofluid considering Joule heating and viscous dissipation. International Journal of Heat and Mass Transfer, 2018, 120, 540-551.

[9] M. J. Uddin, O. A. Bég, N. Amin, Hydromagnetic transport phenomena from a stretching or shrinking nonlinear nanomaterial sheet with Navier slip and convective heating: a model for bio-nano-materials processing. Journal of Magnetism and Magnetic Materials, 2014, 368, 252-261.

[10] T. Zohra, T. Fatema, M. Jashim Uddin, M. Ismail, A. Izani, O. Anwar Bég, Bioconvective electromagnetic nanofluid transport from a wedge geometry: Simulation of smart electroconductive bio-nanopolymer processing. Heat Transfer-Asian Research, 2018, 47(1), 231-250.

[11] R. Kumar, S. Sood, M. Sheikholeslami, S. A. Shehzad, Nonlinear thermal radiation and cubic autocatalysis chemical reaction effects on the flow of stretched nanofluid under rotational oscillations. Journal of colloid and interface science, 2017, 505, 253-265.

[12] M. J. Uddin, Y. Alginahi, O. A. Bég, M. N. Kabir, Numerical solutions for gyrotactic bioconvection in nanofluid-saturated porous media with Stefan blowing and multiple slip effects. Computers \& Mathematics with Applications, 2016, 72(10), 2562-2581. 
[13] R. Kumar, S. Sood, Numerical analysis of stagnation point nonlinear convection flow through porous medium over a shrinking sheet. International Journal of Applied and Computational Mathematics, 2017, 3(2), 971-985.

[14] M. J. Uddin, O. A. Bég, M. N. Uddin, Energy conversion under conjugate conduction, magneto-convection, diffusion and nonlinear radiation over a non-linearly stretching sheet with slip and multiple convective boundary conditions. Energy, 2016, 115, 1119-1129.

[15] R. Kumar, S. Sood, S. A. Shehzad, M. Sheikholeslami, Numerical modeling of time-dependent bio-convective stagnation flow of a nanofluid in slip regime. Results in physics, 2017, 7, 3325-3332.

[16] M. J. Uddin, W. A. Khan, A. I. Ismail, MHD free convective boundary layer flow of a nanofluid past a flat vertical plate with Newtonian heating boundary condition. PLoS One, 2012, 7(11), e49499.

[17] R. Kumar, S. Sood, Effect of quadratic density variation on mixed convection stagnation point heat transfer and MHD fluid flow in porous medium towards a permeable shrinking sheet. Journal of Porous Media, 2016, 19(12), 1083-1097.

[18] 0. Jambal, T. Shigechi, G. Davaa, S. Momoki, Effects of viscous dissipation and fluid axial heat conduction on heat transfer for non-Newtonian fluids in ducts with uniform wall temperature: Part I: Parallel plates and circular ducts, Int. Commun. heat mass Transf., 2005, 32(9), 1165-1173.

[19] O. Ojjela, N. N. Kumar, Chemically reacting micropolar fluid flow and heat transfer between expanding or contracting walls with ion slip, Soret and Dufour effects, Alexandria Engineering Journal, 2016, 55(2), 1683-1694.

[20] Y. L. Chen, K. Q. Zhu, Couette-Poiseuille flow of Bingham fluids between two porous parallel plates with slip conditions, J Nonnewton Fluid Mech., 2008, 153(1), 1-11.

[21] D. Srinivasacharya, N. Srinivasacharyulu, O. Odelu, Flow and heat transfer of couple stress fluid in a porous channel with expanding and contracting walls, Int Commun Heat Mass Transf., 2009, 36(2), 180-185.

[22] O. D. Makinde, T. Chinyoka, MHD transient flows and heat transfer of dusty fluid in a channel with variable physical properties and Navier slip condition, Comput Math with Appl., 2010, 60(3), 660-669.

[23] Zaheer Abbas, Mariam Sheikh, Numerical study of homogeneous-heterogeneous reactions on stagnation point flow of ferrofluid with non-linear slip condition. Chinese Journal of Chemical engineering, 2017, 25, 11-17.

[24] P. A. Thompson, S. M. Troian, A general boundary condition for liquid flow at solid surfaces, Nature, 1997, 389, 360-362.

[25] X. Y. Si, X. H. Si, L. C. Zheng, X. X. Zhang, Homotopy analysis solution for micropolar fluid flow through porous channel with expanding or contracting walls of different permeabilities, Appl Math Mech (English Ed.), 2011, 32(7), 859-874.

[26] S. Xinhui, Z. Liancun, C. Xuehui, Z. Xinxin, C. Limei, L. Min, The effects of slip velocity on a micropolar fluid through a porous channel with expanding or contracting walls, Comput Methods Biomech Biomed Engin., 2014, 17(4), 423-432.

[27] G. S. Beavers, D. D. Joseph, Boundary conditions at a naturally permeable wall, Journal of fluid mechanics, 1967, 30(01), 197207.

[28] M. Shojaeian, K. Ali, Convective heat transfer and entropy generation analysis on Newtonian and non-Newtonian fluid flows between parallel-plates under slip boundary conditions, International Journal of Heat and Mass Transfer, 2014, 70, 664-673.

[29] N. Ahmed, S.T. Mohyud-Din, S. M. Hassan, Flow and heat transfer of nanofluid in an asymmetric channel with expanding and contracting walls suspended by carbon nanotubes: a numerical investigation, Aerosp Sci Technol., 2016, 48, 53-60.

[30] J. G. Oldroyd, On the formulation of rheological equations of state, In: Proceedings of the Royal Society of London A: Mathematical, Physical and Engineering Sciences, 1950, 200, 523-541.

[31] J. G. Oldroyd, Some steady flows of the general elasticoviscous liquid, In: Proceedings of the Royal Society of London A: Mathematical, Physical and Engineering Sciences, 1965, 283, 15-133.

[32] Stefan Zahorski, Mechanics of viscoelastic fluids, Vol. 2. Springer, 1982.

[33] Nasir Ali, Sami Ullah Khan, Muhammad Sajid, Zaheer Abbas, Flow and heat transfer of hydromagnetic Oldroyd-B fluid in a channel with stretching walls, Nonlinear Engineering, 2016, 59 (2), 73-79.

[34] Si Xinhui, Zheng Liancun, Zhang Xinxin, Si Xinyi and Li Min. Asymmetric viscoelastic flow through a porous channel with expanding or contracting walls: a model for transport of biological fluids through vessels, Computer methods in biomechanics and biomedical engineering, 2014, 17(6), 623-631.

[35] M. M. Bhatti, M. A. Abbas, Simultaneous effects of slip and MHD on peristaltic blood flow of Jeffrey fluid model through a porous medium, Alexandria Engineering Journal, 2016, 55(2), 1017-1023.

[36] Sharma, Kalpna, Sumit Gupta, Viscous dissipation and thermal radiation effects in MHD flow of Jeffrey nanofluid through impermeable surface with heat generation/absorption. Nonlinear Engineering, 2017, 6(2), 153-166.

[37] V. Singh, Shweta Agarwal, Robert A. Van Gorder, Closed-form exact solution for the heat transfer due to a second grade fluid over a shrinking sheet, Nonlinear Engineering, 2013, 2, 113119.

[38] K. Chand, R. Kumar, Hall effect on heat and mass transfer in the flow of oscillating viscoelastic fluid through porous medium with wall slip conditions. Indian Journal of Pure and Applied Physics, 2012, 50, 149-155.

[39] R. Kumar, S. Sood, S. A. Shehzad, M. Sheikholeslami, Radiative heat transfer study for flow of non-Newtonian nanofluid past a Riga plate with variable thickness. Journal of Molecular Liquids, 2017, 248, 143-152.

[40] W. A. Khan, M. J. Uddin, A. M. Ismail, Free convection of nonNewtonian nanofluids in porous media with gyrotactic microorganisms. Transport in porous media, 2013, 97(2), 241252.

[41] T. Hayat, M. Ijaz Khan, M. Waqas, A. Alsaedi, T. Yasmeen, Diffusion of chemically reactive species in third grade flow over an exponentially stretching sheet considering magnetic field effects, Chinese Journal of Chemical engineering, 2017, 25(3), 257-263.

[42] P. V. Satya Narayana, D. Harish Babu Numerical study of MHD heat and mass transfer of a Jeffrey fluid over a stretching sheet with chemical reaction and thermal radiation, Journal of the Taiwan Institute of Chemical Engineers, 2016, 59, 18-25. 
[43] Stanley Middleman, Fundamentals of polymer processing, McGraw-Hill College, 1977.

[44] A. Alizadeh-Pahlavan, S. Kayvan, On the use of homotopy analysis method for solving unsteady MHD flow of Maxwellian fluids above impulsively stretching sheets, Communications in Nonlinear Science and Numerical Simulation, 2009, 14(4), 1355-1365.

[45] Swati Mukhopadhyay, Heat transfer analysis of the unsteady flow of a Maxwell fluid over a stretching surface in the presence of a heat source/sink, Chinese Physics Letters, 2012, 29(5), 054703.

[46] K. Vajravelu, K. V. Prasad, A. Sujatha, N. G. Chiu-on, MHD flow and mass transfer of chemically reactive upper convected Maxwell fluid past porous surface, Appl Math Mech (English Ed.), 2012, 33(7), 899-910.

[47] T. Hayat, M. Awais, Three dimensional flow of upper convected Maxwell (UCM) fluid, International Journal for Numerical Methods in Fluids, 2011, 66(7), 875-884.

[48] M. Bilal, M. Sagheer, S. Hussain, Three dimensional MHD upper-convected Maxwell nanofluid flow with nonlinear radiative heat flux, Alexandria Engineering Journal, 2017, http://dx.doi.org/10.1016/j.aej.2017.03.039.

[49] S. K. Nandy, Unsteady flow of maxwell fluid in the presence of nanoparticles towards a permeable shrinking surface with navier slip, Journal of the Taiwan Institute of Chemical Engineers, 2015, 52, 22-30.

[50] J. J. Choi, Zvi Rusak, J. A. Tichy, Maxwell fluid suction flow in a channel, Journal of non-newtonian fluid mechanics, 1999, 85(2), 165-187.

[51] T. Hayat, Z. Abbas, Channel flow of a Maxwell fluid with chemical reaction, Zeitschrift für angewandte Mathematik und Physik, 2008, 59(1), 124-144.

[52] T. Hayat, R. Sajjad, Z. Abbas, M. Sajid, A. A. Hendi, Radiation effects on MHD flow of Maxwell fluid in a channel with porous medium, Int J Heat Mass Transf., 2011, 54(4), 854-862.

[53] O. A. Bég, O.D. Makinde Viscoelastic flow and species transfer in a Darcian high-permeability channel, Journal of Petroleum Science and Engineering, 2011, 76(3), 93-99.

[54] P. G. Saffman, On the boundary condition at the surface of a porous medium, Studies in Applied Mathematics, 1971, 50(2), 93-101.

[55] Andro Mikelic, Willi Jäger, On the interface boundary condition of Beavers, Joseph, and Saffman, SIAM Journal on Applied Mathematics, 2000, 60(4), 1111-1127.

[56] I. Proudman, K. Johnson, Boundary-layer growth near a rear stagnation point. Journal of Fluid Mechanics, 1962, 12(2), 161168.

[57] G. W. Sutton, A. Sherman, Engineering magnetohydrodynamics, Courier Dover Publications, 2006.

[58] Jaan Kiusalaas, Numerical Methods in Engineering with MATLAB, Cambridge University press, 2005.

\section{A Appendix}

Shooting algorithm:

The system of equations are solved using the shooting algorithm. The algorithm consists of Runge Kutta 4th order and Newton Raphson functions. The initial value problem (20-21) is integrated till $\lambda=1$. It can be clearly seen that there are 6 variables as1, as2... as 6 in the initial vector (20) for RK integration and there are exactly 6 boundary conditions at ' 1 '. The values at 1 which are obtained through RK4 method are substituted in these boundary conditions to obtain 6 nonlinear algebraic equations. However the implementation of the above idea needs initial vector for RK4 in terms numbers rather than symbols. Hence Newton Raphson is a good choice to solve the system of coupled nonlinear equations.

Basic steps:

1. Initialisation of the parameters: It contains 14 main parameters with two boundary parameters XSTART and XSTOP stated in the main code.

2. Initialisation of initial vector of newton raphson vector 'as' and therefore initial vector of RK4 in the function 'inCond(as)'.

3. The governing system of equations are stored in dEqs.

4. The boundary conditions are written in Residual function which is called in Newton Raphson (NR) program. The NR function uses the Jacobian function to find the accurate root.

5. The apt values of the assumed slopes are obtained through Newton Raphson program such that the boundary conditions are satisfied by calling Residual function. These values are stored in 'as' by replacing the original guess.

6. The final 'as' is substituted in the RK4 initial vector to obtain the accurate solution.

The basic code for shooting algorithm has been presented by Jaan Kiusalaas [58]. This code has been adopted and modified for coupled nonlinear equations with multiple functions of $\lambda$. 